\title{
Three Degree-of-freedom Modeling of the Random Fluctuation Arising in Human-bicycle Balance* $^{*}$
}

\author{
Katsutoshi Yoshida $^{\dagger}$, Mikinori ITO $^{\dagger}$, Hayate KaIdo $^{\dagger}$ and Yoshikazu YamanaKA ${ }^{\dagger}$
}

\begin{abstract}
A novel three degree-of-freedom (DOF) fluctuation model that accurately reproduces the probability density functions (PDFs) of human-bicycle balance motions is proposed. We experimentally obtain the time series of the roll angular displacement, wheel's lateral displacement, steering angular displacement, and each velocity of them. Constructing the PDFs of these time series as training data, we identify the model parameters through the use of particle swarm optimization (PSO) that minimizes the squared residuals between the experimental participants' PDFs and those simulated by our model. Over $97 \%$ PDF fitnesses were obtained for all participants, indicating that our proposed model can successfully simulate the measured human PDFs.
\end{abstract}

\section{Introduction}

Bicycles provide a useful means of short-distance transportation and their utilization is expected to contribute to building a healthy and environmentally friendly society[1]. However, at least on Japanese roads, bicycle transportation is not always safe due to collisions with automobiles. The Japan National Police Agency reported that over $83 \%$ of bicycle accidents in Japan in the last ten years have involved automobiles[2]. To avoid such accidents, autonomous vehicle technology will play an important role; if it can predict bicycle motions, the resulting self-driving cars may reduce such accidents. For this purpose, accurate simulation models of bicycle motions are required and they should be provided as simply as possible for the potential use of electronic control units in self-driving cars.

Bicycle motions with human riders in traffic seem to be classified into two types: voluntary and involuntary. The former comprises purposeful motions such as right and left turning at a street intersection. Google[3] reported that their sensors can detect a cyclists' hand signals as an indication of an intention to make a turn or shift over. On

* Manuscript Received Date: February 24, 2021

* The material of this paper was partially presented at the 52nd ISCIE International Symposium on Stochastic Systems Theory and Its Applications (SSS'20) which was held in October, 2020.

$\dagger$ Department of Mechanical and Intelligent Engineering, Utsunomiya University; 7-1-2 Yoto, Utsunomiya, Tochigi 321-8585, JAPAN

Key Words: human-bicycle balance, stochastic model, identification, probability density function, particle swarm optimization. the other hand, the latter comprises unconscious motions such as human fluctuated balance motions, which have already been found universally in human quiet standing[4-6], human stick balancing[7,8], visuomotor tracking[9-11], and so on.

In this study, we propose a new three DOF stochastic model that accurately reproduces the PDFs of human-bicycle motions with respect to the roll angular displacement, wheel's lateral displacement, steering angular displacement, and respective velocities. The proposed model in this study provides a larger DOF extension to our previous single DOF model[12] with respect to the roll angle only and is expected to provide a key technology of simulating the bicycle running paths. To this end, we have conducted an experiment in which each human participant rides a bicycle on bicycle-trainer rollers, allowing it to move without rolling or yawing constraints. During this experiment, we measure the time-series of the abovementioned displacements and velocities and obtain their PDFs. Next, we design our proposed human-bicycle model as a carted inverted pendulum mechanism controlled by our human controller model, which was successfully used in our previous studies to simulate random human fluctuations during visuomotor tracking[11] and our previous single DOF human-bicycle experiment[12]. We then identify the model parameters based on the measured PDFs as training data, using PSO to minimize the squared residuals (SRs) between the measured PDFs and those simulated by our proposed human-bicycle model. It will be shown that our proposed model successfully reproduces the measured PDFs with SR fitnesses of over $97 \%$.

Our approach mentioned above sharply contrasts with other studies in the fields of autonomous 


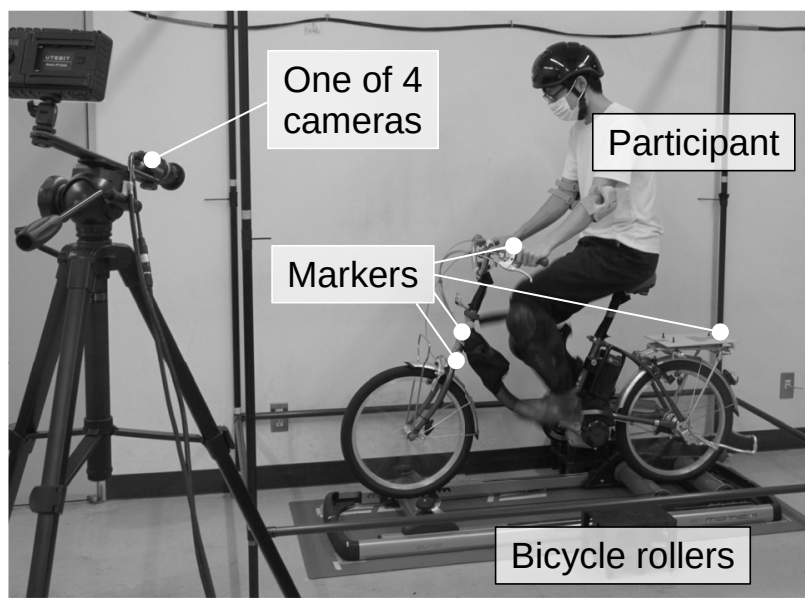

Fig. 1 Photograph of our experimental device and a human participant

or unmanned bicycle-control systems[13-21] because their models have been deterministic and not designed to have randomly fluctuating terms. Our approach also differs from Google's study on voluntary bicycle motions, as mentioned above[3]. Although there has been another study on stochastic modeling of bicycle fluctuated motion[22], it addressed the large-scale bicycle running paths, unlike our study, which deals with small fluctuations.

The rest of the paper is structured as follows: Section 2 describes the human-bicycle experiment. In Section 3, our proposed stochastic model is presented. In Sections $\mathbf{4}$ and $\mathbf{5}$, we describe our method of parameter identification and present the results. Section 6 concludes our study.

This paper is an extended version of our conference paper published in[23]. We extend our previous work by showing further experimental data and describing detailed explanations of our experimental method, conditions, and results, as well as those for numerical calculations.

\section{Human-bicycle Balance Experi- ment}

\subsection{Experimental setup and procedure}

Fig. 1 shows a photograph of our experimental device and a human participant. The experimental device consists of five units: a bicycle (BE-ELL03, Panasonic, Japan), a set of bicycle-trainer rollers (EMOTION, Elite, Italy), a set of motion capture cameras (GV500, Library, Japan), a three-dimensional measurement software (Move-tr/3D, Library, Japan), and a computer.

The experimental participants were three healthy males in their early twenties. They were first instructed on the operation of the experimental device, the number of trials, and the duration of each trial. The experiment was performed according to the principles of the Declaration of Helsinki and informed consent was obtained.

In each trial, the participant rode the bicycle on

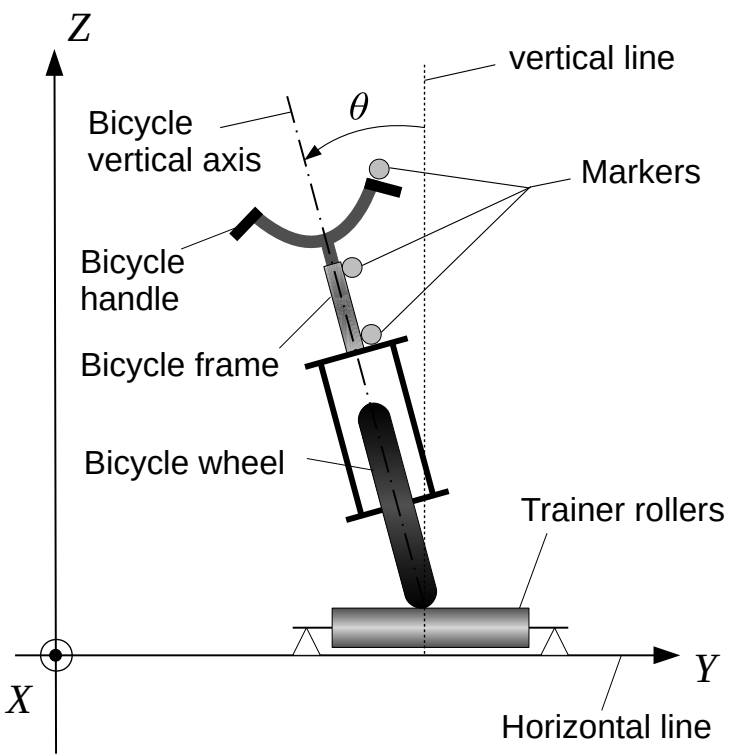

Fig. 2 Schematic front view of the bicycle during the experiment

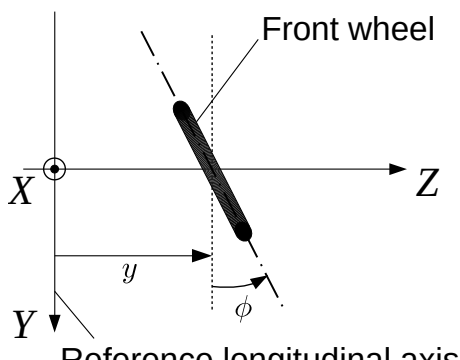

Fig. 3 Schematic top view of the bicycle front wheel during the experiment

the trainer rollers for significantly more than $257 \mathrm{~s}$ at a speed about $12 \mathrm{~km} / \mathrm{h}$. Several practice trials were performed prior to measurement. The air pressure of the tires was set to $680 \mathrm{kPa}$.

The experiment was performed within a quiet room and the running process on the rollers was sufficiently smooth; we suppose that environmental disturbances in this study are negligible.

\subsection{Experimental Data}

During each trial, the time series of the measurement vector,

$$
\begin{aligned}
\boldsymbol{x}(t) & =\left(x_{1}(t), x_{2}(t), \cdots, x_{6}(t)\right)^{T} \\
& :=(\theta(t), \dot{\theta}(t), y(t), \dot{y}(t), \phi(t), \dot{\phi}(t))^{T}
\end{aligned}
$$

(hereafter, $(\cdot)^{T}$ denotes a transpose), was obtained by the motion capture cameras. Here, $\theta$ [rad] is the roll angle from the vertical line to the bicycle's vertical axis, $\dot{\theta}:=d \theta / d t[\mathrm{rad} / \mathrm{s}]$ is the corresponding angular velocity, $y[\mathrm{~m}]$ is the wheel's lateral displacement, $\dot{y}:=$ $d y / d t$ is the corresponding wheel's lateral velocity, $\phi$ is the steering angle from the reference longitudinal axis to the bicycle's longitudinal axis, and $\dot{\phi}:=d \phi / d t$ is the corresponding angular velocity. 

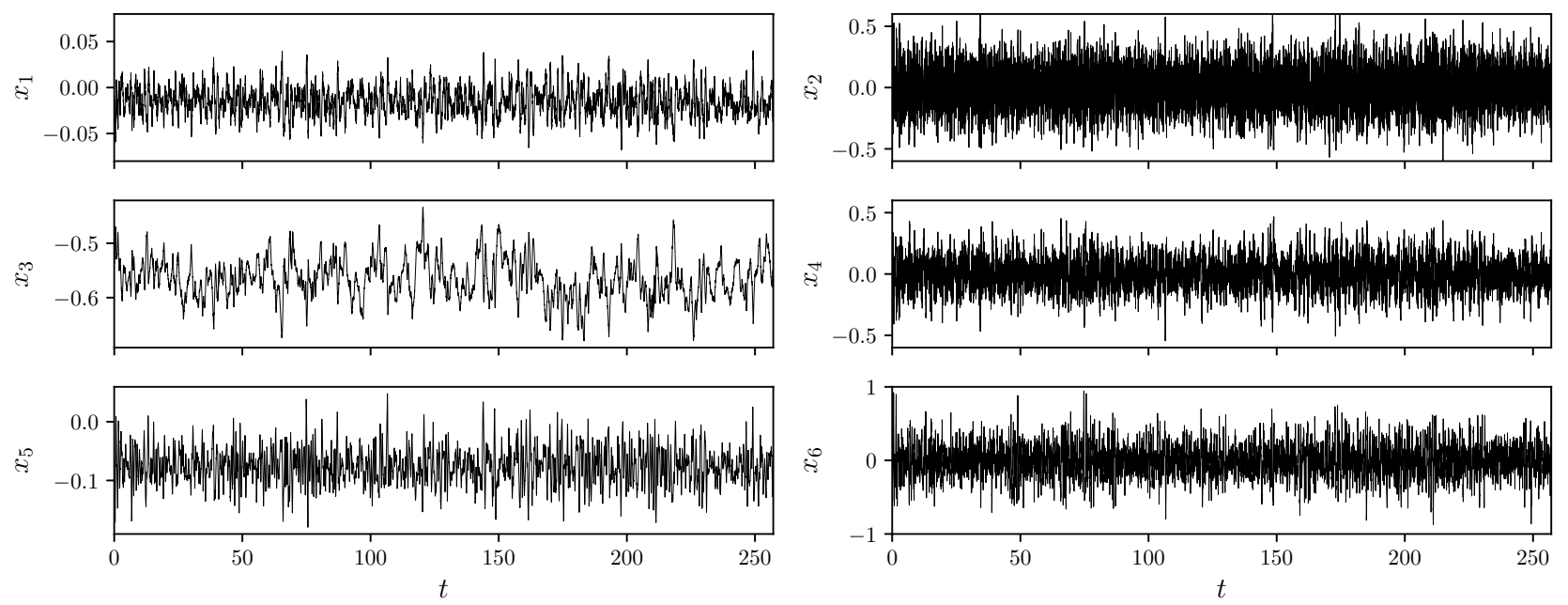

Fig. 4 The measured time series of the human-bicycle balance for $(s, n)=(3,1)$

Fig. 2 schematically shows the definition of the roll angle $\theta$ in the front view of the bicycle and Fig. 3 shows those of the wheel's lateral displacement $y$ and the steering angle $\phi$ in the top view of the bicycle front wheel. The bicycle's vertical and longitudinal axes were nominally determined based on the direction of the bicycle frame and the bicycle handle stem, respectively. The axis of the lateral wheel's displacement were nominally determined based on the direction of the rotation axis of the bicycle-trainer's front roller.

Note that, in our experiment, the rotational origins $\theta=0$ and $\phi=0$ were calibrated to fit the bicycle's vertical parking position; therefore, these do not indicate, respectively, the physical upright equilibrium and the physical straight running position of the unmanned bicycle. In addition, the wheel's lateral origin $y=0$ does not indicate vibrational center of $y$, which center depends on the human riding motions.

Throughout the experiment, $\boldsymbol{x}(t)$ were stored in the computer in the following form:

$$
\begin{gathered}
\left\{\boldsymbol{x}_{\text {hum }}^{(s, n)}\left(t_{0}\right), \cdots, \boldsymbol{x}_{\text {hum }}^{(s, n)}\left(t_{i}\right), \cdots, \boldsymbol{x}_{\text {hum }}^{(s, n)}\left(t_{I-1}\right)\right\}, \\
i=0, \cdots, I-1, s=1, \cdots, S, n=1, \cdots, N,
\end{gathered}
$$

where $t_{i}:=i \Delta t[\mathrm{~s}]$ is a discrete time with a sampling period of $\Delta t, I$ is the length of the time series, $s$ and $S$ are an index and the number of participants, respectively, and $n$ and $N$ are an index and the number of trials, respectively.

For this study, we chose $\Delta t=1 / 70 \mathrm{~s}$ and $I=18001$ to obtain the physical data length $(I-1) \Delta t=257 \mathrm{~s}$. The number of participants was $S=3$ and the number of trials undertaken by each participant was $N=5$.

Fig. 4 shows the measured time series for $(s, n)=$ $(3,1)$, i.e., for the third participant's first trial. Thus, we obtained the time series for all $s=1, \cdots, S$ and $n=1, \cdots, N$. For reference, the result seems to exhibit human specific fluctuations in which large-amplitude spikes intermittently arise among the moderate- amplitude fluctuation process, which have already been recognized as temporal intermittency in the field of nonlinear physics[6-8,24].

As shown in the left column graphs of Fig. 4 , the vibrational centers of $x_{1}:=\theta, x_{3}:=\phi$, and $x_{5}:=y$ are shifted from their nominal origins $(\theta=0, \phi=0$, and $y=0$ ) as mentioned in $\mathbf{2 . 2}$. We therefore statistically evaluate these shifts by identifying them as the mean values of these displacements given by

$$
\begin{array}{r}
E^{(s)}\left[x_{k}\right]:=\frac{1}{N \times I} \sum_{n=1}^{N} \sum_{i=0}^{I-1} x_{k}^{(s, n)}\left(t_{i}\right), \\
\quad s=1, \cdots, S, \quad k=1,3,5,
\end{array}
$$

which is the temporal average of the $s$ th participant's $\theta(t), y(t)$, and $\phi(t)$ further averaged over all of his trials. These mean values are hereafter denoted by $\hat{\theta}^{(s)}, \hat{y}^{(s)}$, and $\hat{\phi}^{(s)}$ (or simply $\hat{\theta}, \hat{y}$, and $\left.\hat{\phi}\right)$, respectively. The resulting values are listed in Table 1 with standard deviations given by

$$
\begin{aligned}
& S D^{(s)}\left[x_{k}\right]=\sqrt{V^{(s)}\left[x_{k}\right]}, \\
& V^{(s)}\left[x_{k}\right]:=\sum_{n=1}^{N} \sum_{i=0}^{I-1} \frac{\left(x_{k}^{(s, n)}\left(t_{i}\right)-E^{(s)}\left[x_{k}\right]\right)^{2}}{N \times I-1}, \\
& \quad s=1, \cdots, S \quad k=1,3,5 .
\end{aligned}
$$

It appears in Table 1 that the $E^{(s)}\left[x_{k}\right](k=1,3,5)$ values are slightly different; these mainly depend on the respective riding forms of the participants.

\subsection{Construction of Measured PDFs}

We obtain $P_{\text {hum }}^{(s, n)}\left(x_{k}\right)$, the PDF with respect to the $k$ th component of the time series in eq. (2) for the sth participant's $n$th trial. This was achieved by normalizing the histogram of the $k$ th component $\left\{x_{k}^{(s, n)}\left(t_{i}\right)\right\}_{i=0}^{I-1}$ with bin width $\left(\overline{x_{k}}-\underline{x_{k}}\right) / N_{\text {bin }}$. Here, $N_{\text {bin }}$ is the number of histogram bins and $\overline{x_{k}}$ and $\underline{x_{k}}$ are the upper and lower limits of $x_{k}$, respectively.

The resulting $P_{\text {hum }}^{(s, n)}\left(x_{k}\right)$ is averaged over all trials $n=1, \cdots, N$ to obtain the $s$ th participant's PDF as 

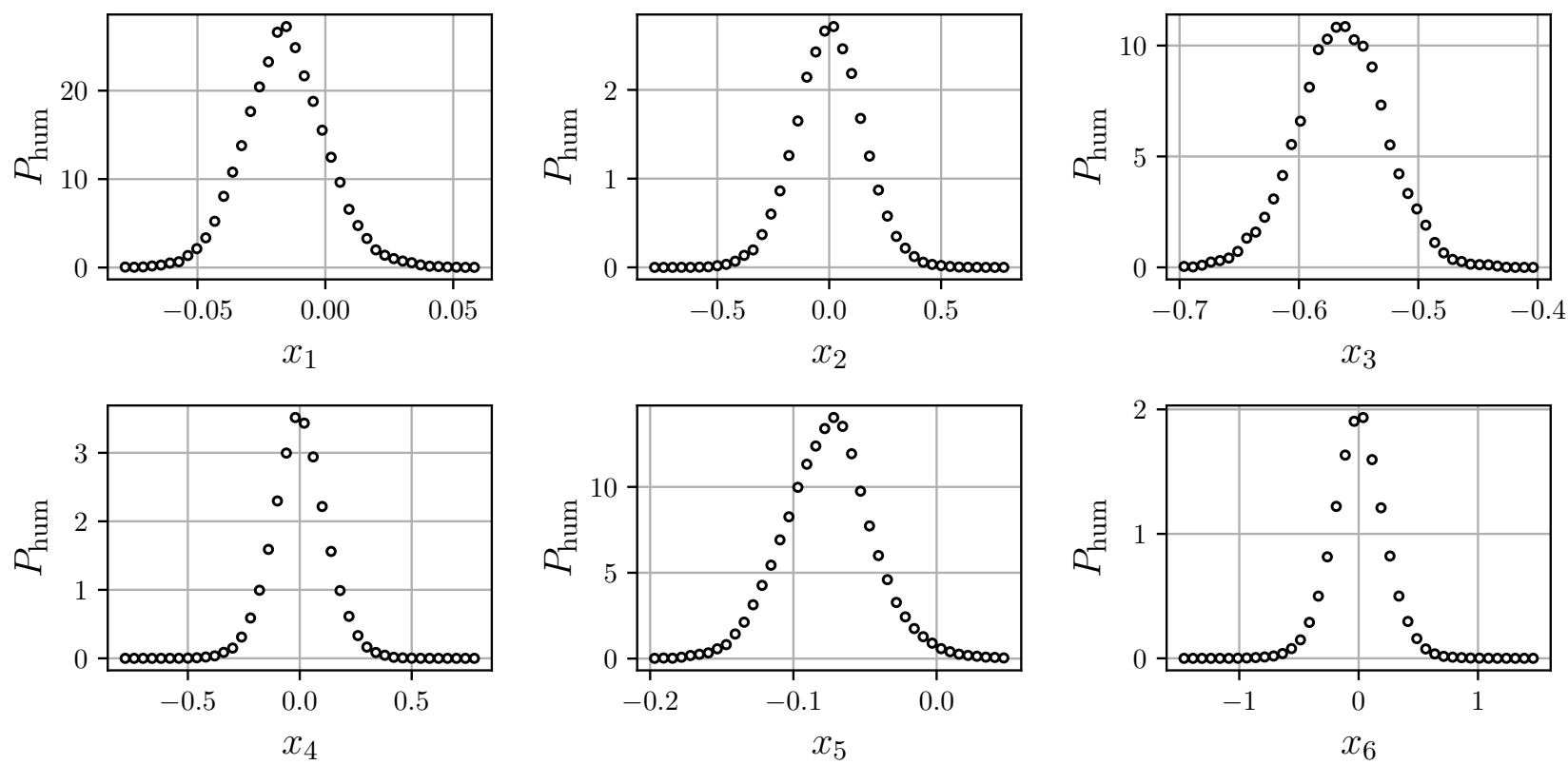

Fig. 5 The measured PDFs $P_{\text {hum }}^{(s)}\left(x_{k}\right), k=1, \cdots, 6$ from the third participant $(s=3)$

Table 1 Time series statistics of the roll angle $x_{1}$, wheel's lateral displacement $x_{3}$, and steering angle $x_{5}$ for the $s$ th participant

\begin{tabular}{llccc}
\hline$s$ & & $x_{1}$ & $x_{3}$ & $x_{5}$ \\
\hline 1 & $E^{(s)}[\cdot]$ & $-1.07 \times 10^{-2}$ & $-5.72 \times 10^{-1}$ & $-1.01 \times 10^{-1}$ \\
& $S D^{(s)}[\cdot]$ & $1.88 \times 10^{-2}$ & $3.68 \times 10^{-2}$ & $3.30 \times 10^{-2}$ \\
\hline 2 & $E^{(s)}[\cdot]$ & $-1.86 \times 10^{-3}$ & $-6.51 \times 10^{-1}$ & $-1.60 \times 10^{-1}$ \\
& $S D^{(s)}[\cdot]$ & $2.39 \times 10^{-2}$ & $3.30 \times 10^{-2}$ & $3.99 \times 10^{-2}$ \\
\hline 3 & $E^{(s)}[\cdot]$ & $-1.56 \times 10^{-2}$ & $-5.64 \times 10^{-1}$ & $-7.59 \times 10^{-2}$ \\
& $S D^{(s)}[\cdot]$ & $1.61 \times 10^{-2}$ & $3.70 \times 10^{-2}$ & $3.12 \times 10^{-2}$ \\
\hline
\end{tabular}

$$
P_{\text {hum }}^{(s)}\left(x_{k}\right)=\frac{1}{N} \sum_{n=1}^{N} P_{\text {hum }}^{(s, n)}\left(x_{k}\right), \quad k=1, \cdots, 6 .
$$

We call eq. (5) the measured PDF of the sth participant.

Fig. 5 shows the measured PDF of the third participant $(s=3)$. In this way, we obtained the measured PDFs for all $s=1,2,3$. In this study, we commonly set $N_{\text {bin }}=40$, while $\overline{x_{k}}$ and $x_{k}$ are chosen for each participant to make them cover the respective distribution of experimental data.

In our previous study[12], we performed a humanbicycle experiment quite similar to that in this study and statistically guaranteed that the human-bicycle roll angular motion $x_{1}(t)$ and $x_{2}(t)$ yielded typical probability distributions which significantly differ from Gaussian ones in terms of Kolmogorov-Smirnov (KS) statistical-hypothesis-testing[12]. Since the state variables added in this study, $x_{3}(t), \cdots, x_{6}(t)$, are mechanically coupled with the non-Gaussian $x_{1}(t)$ and $x_{2}(t)$, they are supposed to be non-Gaussian as well. Unfortunately, as rigorous formulation of multidimensional KS testing seems to be still an open problem, statistical testing of our six-dimensional data will be a future problem.
Additionally, note that the double-indexing of $P_{\text {hum }}^{(s, n)}\left(x_{k}\right)$ to store the measured PDF is intended for potential use in our future work on the effects of the number of trials $n$ although the numerical calculation of $P_{\text {hum }}^{(s)}\left(x_{k}\right)$ by eq. (5) can equivalently be replaced with calculating the histogram from the union of $s$ th participant's data.

\section{Fluctuation Model of the Human-bicycle Balance}

In this section, we propose a new three DOF stochastic model that allows us to reproduce the measured PDFs obtained above as simply as possible.

\subsection{A Human-bicycle Fluctuation Model}

We model the human-bicycle mechanics by a carted inverted pendulum. Based on the time series statistics in Table 1, we also assume that the roll angle $x_{1}=\theta$ about the equilibrium and steering angle $x_{5}=\phi$ about the straight running is sufficiently small; the maximal three standard deviation indicates $3 \times \max _{s} S D^{(s)}\left[x_{1}\right] \approx 7.17 \times 10^{-2} \mathrm{rad}\left(\right.$ or $4.10^{\circ} \mathrm{deg}$ ) and $3 \times \max _{s} S D^{(s)}\left[x_{5}\right] \approx 1.19 \times 10^{-1} \mathrm{rad}$ (or $6.81^{\circ}$ deg). Therefore, we model the bicycle's motion by 
a linearized carted inverted pendulum of the form:

$$
\left\{\begin{array}{l}
\ddot{y}+\ddot{\theta}-g(\theta-\hat{\theta})+c_{\theta} \dot{\theta}=0 \\
\ddot{y}+c_{y} \dot{y}+c_{\phi} \dot{\phi}=V \dot{\phi}
\end{array}\right.
$$

This can be transformed, using the state vector $\boldsymbol{x}=$ $\left(x_{1}, \cdots, x_{6}\right)^{T}:=(\theta, \dot{\theta}, y, \dot{y}, \phi, \dot{\phi})^{T}$, into the state-space form:

$$
\left\{\begin{array}{l}
\dot{x}_{1}=x_{2}, \dot{x}_{2}=-V x_{6}+c_{y} x_{4}+g\left(x_{1}-\hat{\theta}\right)-c_{\theta} x_{2} \\
\dot{x}_{3}=V\left(x_{5}-\hat{\phi}\right), \dot{x}_{4}=V x_{6}-c_{y} x_{4}-c_{\phi} x_{6} \\
\dot{x}_{5}=x_{6}, \dot{x}_{6}=u(t)
\end{array}\right.
$$

where $u(t)$ is a human input, $g:=9.81 \mathrm{~m} / \mathrm{s}^{2}$ is gravitational acceleration, $c_{\theta}, c_{y}$, and $c_{\phi}$ are viscous dampings, and $V$ is a bicycle speed.

The human input $u(t)$ is specified to simulate the human fluctuation during human-bicycle motion. As successfully demonstrated in our previous studies[11,12], some human fluctuations can be accurately simulated by the following state-feedback mechanism:

$$
\begin{aligned}
u(t)= & F_{1}\left(x_{1}-\hat{\theta}\right)+F_{2} x_{2}+F_{3}\left(x_{3}-\hat{y}\right)+F_{4} x_{4} \\
& +\mu\left\{1+\sigma_{1} \xi_{1}(t)\right\}\left(x_{5}-\hat{\phi}\right)+F_{6} x_{6}+\sigma_{2} \xi_{2}(t) .
\end{aligned}
$$

Here, $F_{1}, \cdots, F_{4}, F_{6}$ are deterministic gains and $\xi_{1}(t)$ and $\xi_{2}(t)$ are independent white Gaussian noises with zero mean and unit variance. Thus, $\mu\left\{1+\sigma_{1} \xi_{1}\right\}$ represents a random proportional gain with mean $\mu$ and variance $\left(\mu \sigma_{1}\right)^{2}$ while $\sigma_{2} \xi_{2}(t)$ represents an additive random perturbation.

Finally, we substitute eq. (8) into eq. (7) and propose a human-bicycle fluctuation model with the eleven-dimensional parameter vector:

$$
\begin{aligned}
\boldsymbol{p} & =\left(p_{1}, \cdots, p_{11}\right) \\
& :=\left(F_{1}, F_{2}, F_{3}, F_{4}, F_{6}, c_{\theta}, c_{y}, c_{\phi}, \mu, \sigma_{1}, \sigma_{2}\right) \in \mathbb{R}^{11},
\end{aligned}
$$

where $\mathbb{R}^{n}$ denotes $n$-dimensional real space. The parameter vector $\boldsymbol{p}$ parameterizes the random dynamical properties of the human-bicycle motion of each participant in terms of classical mechanics as well as control engineering.

The remarkable feature of our proposed model (eq. (7) and eq. (8)) is that it is driven by the random noises $\xi_{1}$ and $\xi_{2}$ only, which simulate human endogenous fluctuations mainly due to hemodynamics and cardiorespiratory dynamics[25]. This means that, in our model identification problem, the input data $\left(\xi_{1}\right.$ and $\left.\xi_{2}\right)$ is experimentally unavailable as timeseries data. Instead, in this study, we mathematically assume probability distributions of the input data and experimentally measure those of the output data $P_{\text {hum }}^{(s)}\left(x_{k}\right)$, which is compared with the simulated output data $P_{\operatorname{sim}}\left(x_{k} ; \boldsymbol{q}, \boldsymbol{p}\right)$ as described in the next section.

\subsection{Calculation of Simulated PDFs}

Using given $\boldsymbol{q}:=(\hat{\theta}, \hat{y}, \hat{\phi})$ and $\boldsymbol{p}$, we obtain $N^{\prime}$ samples of the stationary numerical solution of eq. (7) and eq. (8) as

$$
\left\{\boldsymbol{x}_{A}^{\left(n^{\prime}\right)}\left(t_{i} ; \boldsymbol{q}, \boldsymbol{p}\right)\right\}_{i=0}^{I-1}, \quad n^{\prime}=1, \cdots, N^{\prime},
$$

by means of a fourth-order Runge-Kutta-Gill method with time step $\Delta t=10^{-2} \mathrm{~s}$. To generate these samples, $N^{\prime}$ different sequences of normal pseudorandom numbers $[26]$ :

$$
\left\{\nu_{i}^{\left(n^{\prime}\right)}\right\}_{i=0}^{I-1}, \quad n^{\prime}=1, \cdots, N^{\prime}
$$

are used to simulate the independent white Gaussian noises $\xi_{1}(t)$ and $\xi_{2}(t)$ by

$$
\xi_{l}\left(t_{i}\right) \approx \nu_{i}^{\left(n^{\prime}\right)}(\Delta t)^{-1 / 2}, \quad l=1,2
$$

where $(\Delta t)^{-1 / 2}$ is the numerical factor required for integrating stochastic differential equations[27].

From the $k$ th component of the simulated time series in eq. (10), we construct the $n^{\prime}$ th sample's PDF $P_{\text {sim }}^{\left(n^{\prime}\right)}\left(x_{k} ; \boldsymbol{q}, \boldsymbol{p}\right)$ using the same procedure and conditions as applied for deriving the measured PDF in 2.3. We also take the average $P_{\text {sim }}^{\left(n^{\prime}\right)}\left(x_{k} ; \boldsymbol{q}, \boldsymbol{p}\right)$ over all samples by

$$
\begin{array}{r}
P_{\mathrm{sim}}\left(x_{k} ; \boldsymbol{q}, \boldsymbol{p}\right)=\frac{1}{N^{\prime}} \sum_{n^{\prime}=1}^{N^{\prime}} P_{\mathrm{sim}}^{\left(n^{\prime}\right)}\left(x_{k} ; \boldsymbol{q}, \boldsymbol{p}\right), \\
k=1,2, \cdots, 6 .
\end{array}
$$

We call eq. (13) the simulated PDFs for $\boldsymbol{q}$ and $\boldsymbol{p}$, which are to be compared with the measured $P_{\text {hum }}^{(s)}\left(x_{k}\right)$.

\section{Method of Parameter Identifica- tion}

In this section, we formulate the identification problem of an unknown parameter vector $\boldsymbol{p}$ of our proposed model. However, as pointed out in 3.1, our identification problem suffers from the lack of input time-series and thus time-series-based approaches are not applicable to our problem. Instead, we here formulate a PDF-based identification approach.

\subsection{Parameter Identification Problem}

We solve the optimization problem

$$
\underset{\boldsymbol{p}}{\operatorname{Minimize}} C(\boldsymbol{p})
$$

with the cost function

$$
\begin{aligned}
C(\boldsymbol{p}) & :=\sum_{k=1}^{6} a_{k} C_{k}(\boldsymbol{p}), \quad a_{k}>0, \\
C_{k}(\boldsymbol{p}) & :=\frac{\int_{\underline{x_{k}}}^{\overline{x_{k}}}\left\{P_{\mathrm{sim}}\left(x_{k} ; \boldsymbol{q}, \boldsymbol{p}\right)-P_{\mathrm{hum}}^{(s)}\left(x_{k}\right)\right\}^{2} d x_{k}}{\int_{\underline{x_{k}}}^{\overline{x_{k}}}\left\{P_{\mathrm{hum}}^{(s)}\left(x_{k}\right)\right\}^{2} d x_{k}} .
\end{aligned}
$$


Here, $a_{k}$ are weight coefficients and $C_{k}(\boldsymbol{p})$ is the integral of the SR between the simulated $P_{\text {sim }}\left(x_{k} ; \boldsymbol{q}, \boldsymbol{p}\right)$ and the measured $P_{\text {hum }}^{(s)}\left(x_{k}\right)$, as normalized by the integral of the squared $P_{\text {hum }}^{(s)}\left(x_{k}\right)$. Therefore, this cost function evaluates the PDF's reproduction error and satisfies $C(\boldsymbol{p})=0$ if $P_{\text {sim }}\left(x_{k} ; \boldsymbol{q}, \boldsymbol{p}\right)=P_{\text {hum }}^{(s)}\left(x_{k}\right)$, $k=1, \cdots, 6$.

\subsection{Procedure of PSO}

We employ PSO[28] to solve eq. (14). Consider a swarm of $M$ candidate solutions,

$$
\left\{\boldsymbol{p}^{1}, \boldsymbol{p}^{2}, \cdots, \boldsymbol{p}^{i}, \cdots, \boldsymbol{p}^{M}\right\}, \quad \boldsymbol{p}^{i} \in \mathbb{R}^{11},
$$

which are called particles. Each component of $\boldsymbol{p}^{i}$ is recursively updated by

$$
\left\{\begin{aligned}
v_{j}^{i}(k+1)= & \omega v_{j}^{i}(k)+c_{1} \eta_{1 j}^{i}(k)\left(p b_{j}^{i}(k)-p_{j}^{i}(k)\right) \\
& +c_{2} \eta_{2 j}^{i}(k)\left(g b_{j}(k)-p_{j}^{i}(k)\right), \\
p_{j}^{i}(k+1)= & p_{j}^{i}(k)+v_{j}^{i}(k+1), \quad k=0,1, \cdots, K
\end{aligned}\right.
$$

where $p_{j}^{i}(k)$ denotes the $j$-th component of $\boldsymbol{p}_{i}$ at iteration $k ; v_{j}^{i}(k)$ is the corresponding velocity; $\omega, c_{1}$, and $c_{2}$ are system parameters of PSO; $\eta_{1 j}^{i}(k)$ and $\eta_{2 j}^{i}(k)$ are random numbers independently generated by [26] for each $i, j$, and $k$ with a uniform distribution in $[0,1]$; and $p b_{j}^{i}(k)$ and $g b_{j}(k)$ are the $j$ th components of the vectors $\boldsymbol{p} \boldsymbol{b}_{i}(k)$ and $\boldsymbol{g} \boldsymbol{b}(k) \in \mathbb{R}^{11}$, respectively. $\boldsymbol{p} \boldsymbol{b}_{i}(k)$ is the position of the particle taking the lowest cost among those at $\boldsymbol{p}_{i}(0), \cdots, \boldsymbol{p}_{i}(k)$; this is called the personal best. $\boldsymbol{g} \boldsymbol{b}(k)$ is the position of the particle with the lowest cost among all particles for all iterations up to $k$; this is called the global best. For sufficiently large $K, \boldsymbol{g} \boldsymbol{b}(K)$ is getting close to the optimal solution $\boldsymbol{p}^{*}$.

\section{Identification Results}

\subsection{Identification Condition}

The PSO was employed with $M=2048$ particles and $K=500$ iterations. The PSO system parameters were set to the optimized values $\omega=0.729$ and $c_{1}=$ $c_{2}=1.49445$ as described in[29]. The initial particles $\boldsymbol{p}^{i}(0), \quad i=1, \cdots, M$ were given by random points uniformly distributed within the eleven-dimensional hyperrectangle empirically given by

$$
\begin{aligned}
\boldsymbol{D}_{0}:= & \left\{\left(p_{1}, \cdots, p_{11}\right) \mid-30 \leq p_{1} \leq 0,0 \leq p_{2} \leq 10\right. \\
& -30 \leq p_{3} \leq 0,0 \leq p_{4} \leq 30,-30 \leq p_{5} \leq 0 \\
& 0 \leq p_{6} \leq 15,0 \leq p_{7} \leq 10,0 \leq p_{8} \leq 3 \\
& \left.0 \leq p_{9} \leq 10,0 \leq p_{10} \leq 20,0 \leq p_{11} \leq 5\right\}
\end{aligned}
$$

The weights in eq. (15) were empirically set to $a_{k}=$ 1 for all $k$ by which the cost values were converged within the $K=500$ iterations and the proper fitness was obtained as described in the next section.

The number of the simulated samples in eq. (13) was $N^{\prime}=5$, the same as the number of experimental trials par participant. Hereafter, $\boldsymbol{p}^{(s)}$ denotes the optimized solution $\boldsymbol{g} \boldsymbol{b}(K)$ obtained from the sth participant's data: $P_{\text {hum }}^{(s)}\left(x_{k}\right)$ and $\boldsymbol{q}^{(s)}=$ $\left(\hat{\theta}^{(s)}, \hat{y}^{(s)}, \hat{\phi}^{(s)}\right)$. We also use the notation $P_{\text {sim }}^{(s)}\left(x_{k}\right):=$ $P_{\text {sim }}\left(x_{k} ; \boldsymbol{q}^{(s)}, \boldsymbol{p}^{(s)}\right)$ for the simulated PDFs derived from the $s$ th participant's data.

\subsection{Identification Results}

Table 2 lists the identified vector components of $\boldsymbol{p}^{(s)}$ by PSO for all participants $s=1,2,3$ and the corresponding SR cost value $C\left(\boldsymbol{p}^{(s)}\right)$. The last column indicates the cost value as a PDF fitness value of the form:

$$
\left\{1-C\left(\boldsymbol{p}^{(s)}\right)\right\} \times 100 \%
$$

which indicates the accuracy of our human-bicycle fluctuation model in eq. (7) and eq. (8) in terms of the reproducibility of PDFs. The resulting PDF fitness values indicate that our proposed model in eq. (7) and eq. (8) successfully achieved over 97\% PDF fitness, even in the worst case $(s=3)$.

In Fig. 6, the solid curves indicate our simulated $P_{\text {sim }}^{(s)}\left(x_{k}\right) \quad(k=1, \cdots, 6)$ for $(\mathrm{a})$ the best case $(s=2)$ and (b) the worst case $(s=3)$. The small circles indicate the corresponding $P_{\mathrm{hum}}^{(s)}\left(x_{k}\right)$ measurements. As already indicated by the PDF fitness values in Table 2, our simulated PDFs mostly agree with the measured PDFs even in the worst case $(s=3)$. Furthermore, the best case $(s=2)$ resulted in much better agreement with the measured PDFs.

\section{Conclusion}

In this study, we have constructed a three DOF human-bicycle fluctuation model that reproduces the PDFs of the experimentally measured human-bicycle balance motions.

First, we measured the time series of the roll angular displacement, wheel's lateral displacement, steering angular displacement, and each velocity of the human-bicycle balance motions and constructed their PDFs. Using these PDFs as the training data, we identified the model parameters by PSO, minimizing the SR between the measured PDF from the participant and the simulated PDF by our model. The resulting PDF fitnesses were over 97\%, indicating that the simulated PDFs reproduced the measured ones.

The above result leads to the conclusion that our proposed model can provide a three degree-of-freedom fluctuation model of human-bicycle fluctuations.

In future work, we plan to simulate the bicycle's running paths based on our proposed model to reduce the bicycle accidents; for this purpose, nonlinear analysis of temporal intermittency $[6-8,24]$ specific to the human-bicycle fluctuations will be a critical issue. Furthermore, we plan to apply a semi-analytical method of calculating PDF residuals[30] towards faster calculation of our method. 
Table 2 Identified vector components of $\boldsymbol{p}^{(s)}=\left(p_{1}, \cdots, p_{11}\right)$ and its cost value $C\left(\boldsymbol{p}^{(s)}\right)$ for the $s$ th participant

\begin{tabular}{cccccccc}
\hline$s$ & $p_{1}$ & $p_{2}$ & $p_{3}$ & $p_{4}$ & $p_{5}$ & $p_{6}$ & \\
\hline 1 & 54.28647 & 14.61676 & 4.156761 & 18.44603 & 5.104233 & 9.158946 & \\
2 & 57.28823 & 7.932240 & 2.008461 & 15.50420 & 1.911434 & 6.228664 & \\
3 & 26.99948 & 10.64740 & 1.428514 & 6.469611 & 3.570648 & 10.08310 & \\
\hline$s$ & $p_{7}$ & $p_{8}$ & $p_{9}$ & $p_{10}$ & $p_{11}$ & $C\left(\boldsymbol{p}^{(s)}\right)$ & PDF fitness \\
\hline 1 & 12.61928 & 10.45926 & 36.59455 & 0.8424895 & 1.766464 & $1.510 \times 10^{-2}$ & $98.49 \%$ \\
2 & 9.352436 & 8.206091 & 12.56037 & 0.1545174 & 0.9200015 & $8.749 \times 10^{-3}$ & $99.12 \%$ (best) \\
3 & 10.95418 & 10.43443 & 10.18957 & 1.241405 & 0.5660814 & $2.924 \times 10^{-2}$ & $97.07 \%$ (worst) \\
\hline
\end{tabular}

(a) The best case $(s=2)$
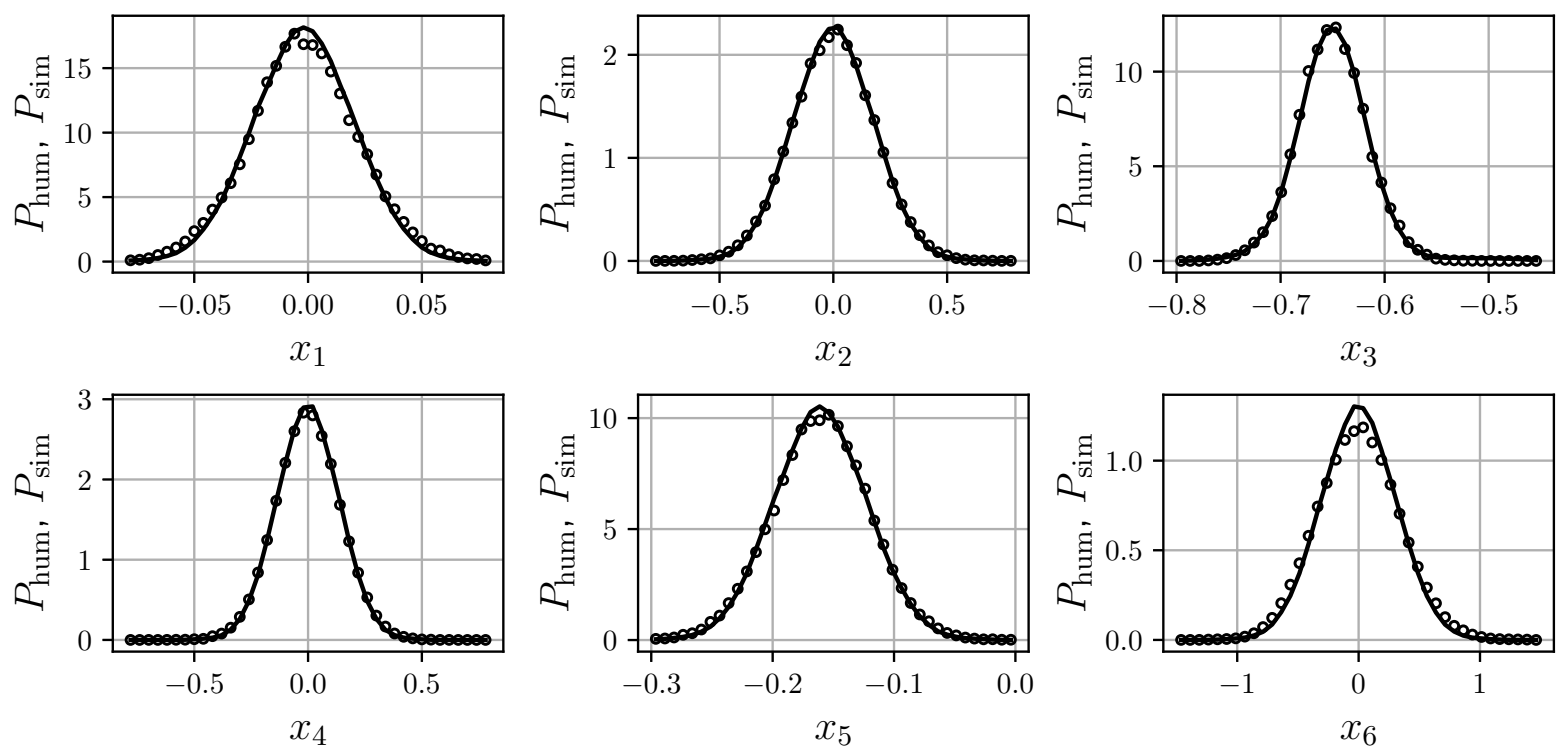

(b) The worst case $(s=3)$
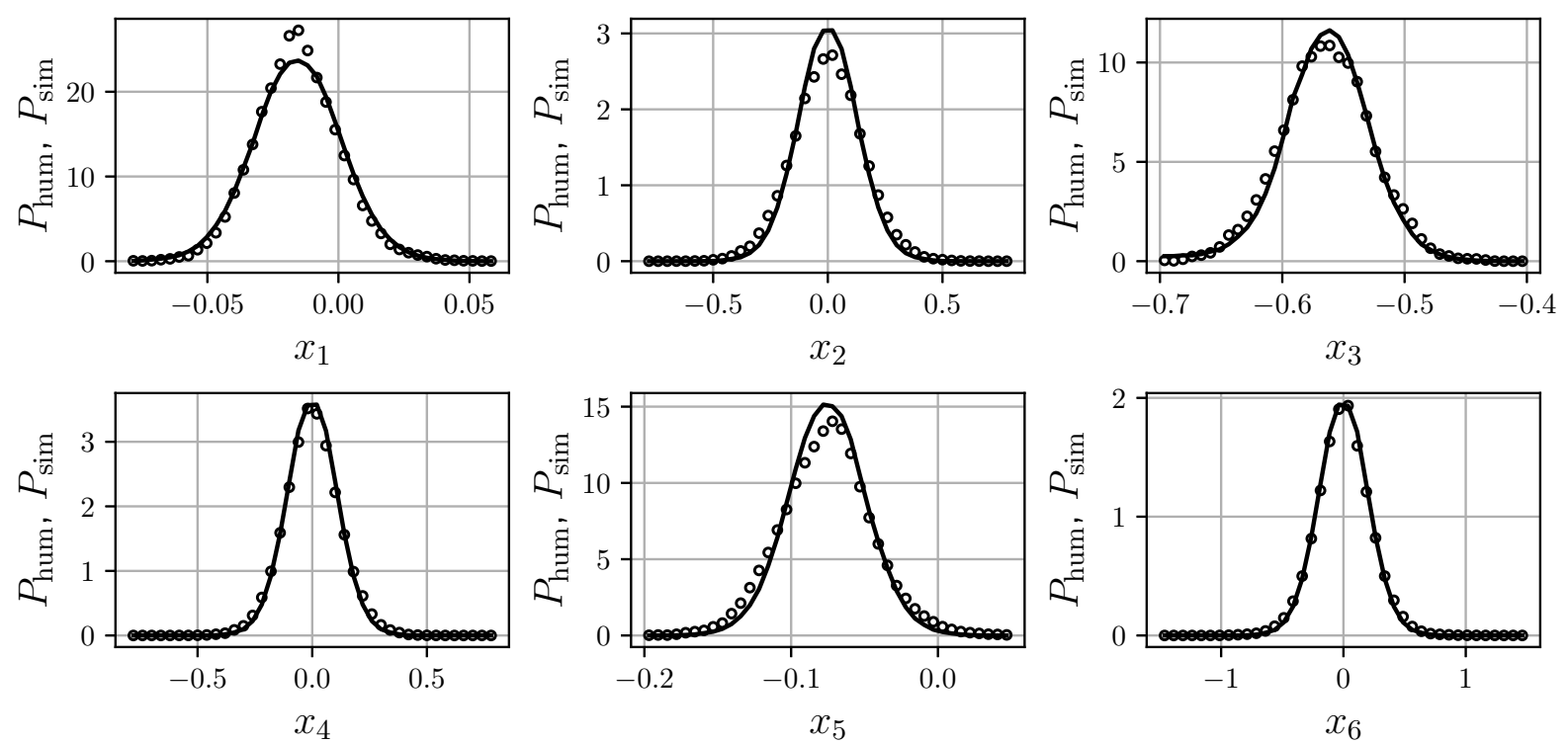

Fig. 6 Comparison between the measured $P_{\text {hum }}^{(s)}\left(x_{k}\right)$ and the simulated $P_{\operatorname{sim}}^{(s)}\left(x_{k}\right), k=1, \cdots, 6$, for (a) the best case $(s=2)$ and (b) the worst case $(s=3)$. The small circles indicate the measured PDFs and the solid curves indicate the simulated PDFs. 


\section{Acknowledgements}

We wish to express our gratitude to the members of the System Dynamics Laboratory at Utsunomiya University for their participation and cooperation as participants in this study. This work was funded by JSPS KAKENHI Grant Number JP18H01391.

\section{References}

[1] Japan Ministry of the Environment: Building a low carbon society; https://www.env.go.jp/earth/ info/pc071211/en.pdf (2007), Accessed: 2019-0311

[2] Japan National Police Agency: Traffic accident statistics annual report; https://www.e-stat. go.jp/en/stat-search/files?lid=000001223644 (2019), Accessed: 2019-03-11

[3] Google self-driving car project optmonthly report; https://www.google.com/selfdrivingcar/ files/reports/report-1016.pdf (2016), Accessed: 2019-03-11

[4] J. J. Collins and C. J. De luca: Random walking during quiet standing; Physical Review Letters, Vol. 73, No. 5, pp. 764-767 (1994)

[5] D. A. Winter, A. E. Patla, F. Prince, M. Ishac and K. Gielo-Perczak: Stiffness control of balance in quiet standing; Journal of Neurophysiology, Vol. 80, No. 3, pp. 1211-1221 (1998)

[6] C. K. Rhea, T. A. Silver, S. L. Hong, J. H. Ryu, B. E. Studenka, C. M. L. Hughes and J. M. Haddad: Noise and complexity in human postural control: interpreting the different estimations of entropy; PloS One, Vol. 6, No. 3, p. e17696 (2011)

[7] J. L. Cabrera and J. G. Militon: On-off intermittency in a human balancing task; Physical Review Letters, Vol. 89, No. 15, pp. 158702:1-4 (2002)

[8] J. L. Cabrera and J. G. Milton: Human stick balancing: Tuning Lévy flights to improve balance control; Chaos, Vol. 14, No. 3, pp. 691-698 (2004)

[9] R. Bormann, J. L. Cabrera, J. G. Milton and C. W. Eurich: Visuomotor tracking on a computer screenan experimental paradigm to study the dynamics of motor control; Neurocomputing, Vol. 58, No. 60 , pp. 517-523 (2004)

[10] K. Yoshida and A. Higeta: Toward stochastic explanation of a neutrally stable delayed feedback model of human balance control; Int. J Innovative Computing, Information and Control, Vol. 8, No. 3, pp. 2249-2259 (2012)

[11] S. Matsumoto, K. Yoshida and M. Sekikawa: Stochastic dynamic modeling of human visuomotor tracking task of an unstable virtual object; Transactions of the Institute of Systems, Control and Information Engineers, Vol. 31, No. 6, pp. 209-219 (2018)

[12] K. Yoshida, K. Sato and Y. Yamanaka: Simple degree-of-freedom modeling of the random fluctuation arising in human-bicycle balance; Applied Sciences, Vol. 9, No. 10, p. 2154 (2019)

[13] N. H. Getz: Control of balance for a nonlinear nonholonomic non-minimum phase model of a bicycle; Proceedings of 1994 American Control Conference - ACC '94, Vol. 1, pp. 148-151 (1994)
[14] N. H. Getz and J. E. Marsden: Control for an autonomous bicycle; Proceedings of 1995 IEEE International Conference on Robotics and Automation, Vol. 2, pp. 1397-1402 (1995)

[15] S. Lee: Self stabilizing strategy in tracking control of unmanned electric bicycle with mass balance; IEEE/RSJ, Int. Conference on Intelligent Robots and Systems, 2002 (2002)

[16] C. K. Chen and T. S. Dao: Fuzzy control for equilibrium and roll-angle tracking of an unmanned bicycle; Multibody System Dynamics, Vol. 15, No. 4, pp. 321-346 (2006)

[17] J. Meijaard, J. M. Papadopoulos, A. Ruina and A. Schwab: Linearized dynamics equations for the balance and steer of a bicycle: a benchmark and review; Proceedings of the Royal Society A: Mathematical, Physical and Engineering Sciences, Vol. 463, No. 2084, pp. 1955-1982 (2007)

[18] C. Hwang, H. Wu and C. Shih: Fuzzy sliding-mode underactuated control for autonomous dynamic balance of an electrical bicycle; IEEE Transactions on Control Systems Technology, Vol. 17, No. 3, pp. 658-670 (2009)

[19] J. D. G. Kooijman, J. P. Meijaard, J. M. Papadopoulos, A. Ruina and A. L. Schwab: A bicycle can be self-stable without gyroscopic or caster effects; Science, Vol. 332, No. 6027, pp. 339-342 (2011)

[20] J. Tan, Y. Gu, C.K. Liu and G. Turk: Learning bicycle stunts; ACM Trans. Graph., Vol. 33, No. 4, pp. 50:1-50:12 (2014)

[21] Y. Kim, H. Kim and J. Lee: Stable control of the bicycle robot on a curved path by using a reaction wheel; Journal of Mechanical Science and Technology, Vol. 29, No. 5, pp. 2219-2226 (2015)

[22] J. Randløv and P. Alstrøm: Learning to drive a bicycle using reinforcement learning and shaping; Proceedings of the Fifteenth International Conference on Machine Learning, ICML '98, pp. 463-471, Morgan Kaufmann Publishers (1998)

[23] M. Ito, H. Kaido, Y. Yamanaka and K. Yoshida: Three degree-of-freedom stochastic modeling of human-bicycle balance fluctuations; Proceedings of the 52nd ISCIE International Symposium on Stochastic Systems Theory and Its Applications (SSS'20) (forthcoming)

[24] K. Yoshida and K. Sato: Chaotic behaviors of nonlinear vibration system with stochastic excitation: Evaluation of scaling structures (in Japanese); Transactions of the Japan Society of Mechanical Engineers Series C, Vol. 65, No. 632, pp. 1305-1312 (1999)

[25] S. Conforto, M. Schmid, V. Camomilla, T. D'Alessio and A. Cappozzo: Hemodynamics as a possible internal mechanical disturbance to balance; Gait $\mathscr{E}$ Posture, Vol. 14, No. 1, pp. 28-35 (2001)

[26] M. Matsumoto and T. Nishimura: Mersenne twister: A 623-dimensionally equidistributed uniform pseudorandom number generator; ACM Trans. Model. Comput. Simul., Vol. 8, No. 1, pp. 3-30 (1998)

[27] D. J. Higham: An algorithmic introduction to numerical simulation of stochastic differential equations; SIAM Review, Vol. 43, No. 3, pp. 525-546 (2012)

[28] Y. Shi and R. Eberhart: A modified particle swarm optimizer; 1998 IEEE International Conference on 
Evolutionary Computation Proceedings. IEEE World Congress on Computational Intelligence, pp. 69-73 IEEE (1998)

[29] R. C. Eberhart and Y. Shi: Comparing inertia weights and constriction factors in particle swarm optimization; Proceedings of the 2000 Congress on Evolutionary Computation, pp. 84-88, IEEE (2000)

[30] K. Yoshida and Y. Yamanaka: Parameter estimation via Fokker-Planck type residual: Application to linear stationary random vibration; Proceedings of MoViC2020 15th International Conference on Motion and Vibration Control, p. 10055 (2019)

\section{Authors}

Katsutoshi Yoshida (Member)

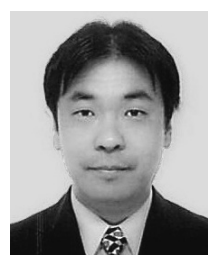

Katsutoshi Yoshida received the Ph.D. degree in Engineering from Utsunomiya University, Utsunomiya, Japan, in 1996. Since 2012, he is Professor at the Graduate School of Engineering, Utsunomiya University. His research interests are in nonlinear dynamics, stochastic mechanics, complex systems, and human dynamics. He is a member of ISCIE and JSME.

\section{Mikinori Іто}

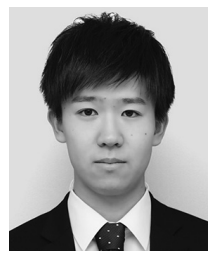

Mikinori Ito received the B.S.(2019) and M.S.(2021) degrees in Engineering from Utsunomiya University, Utsunomiya, Japan. His research interests include human dynamics.

\section{Hayate KAIDO}

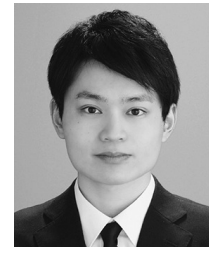

Hayate Kaido received the B.S.(2020) degree in Engineering from Utsunomiya University, Utsunomiya, Japan. His research interests include human dynamics.

\section{Yoshikazu YAMANAKA}

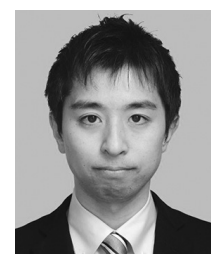

Yoshikazu Yamanaka received the Ph.D. degree in Engineering from Nagaoka University of Technology, Niigata, Japan, in 2017. Since 2017, he is Assistant Professor at the Graduate School of Engineering, Utsunomiya University. His research interests are in evolutionary computation, swarm intelligence, and nonlinear dynamics. $\mathrm{He}$ is a member of IEICE and IEEE. 